\title{
Cartilage and chondrocyte pathology in the mucopolysaccharidoses: The role of glycosaminoglycan-mediated inflammation
}

\author{
Calogera M. Simonaro* \\ Mount Sinai School of Medicine, New York, NY, USA
}

Keywords: Mucopolysaccharidoses, cartilage, inflammation

\section{Introduction}

The mucopolysaccharidoses are a family of genetic diseases each caused by a defect in a lysosomal enzyme responsible for degradation of glycosaminoglycan (GAG) [11]. Failure of effective metabolism results in abnormal storage of GAG fragments, cell injury and death, and inflammatory responses. Because glycosaminoglycans are fundamental in connective tissue structure and function, the MPS disorders are characterized by severe skeletal abnormality including growth failure, abnormal bone structure (dysostosis multiplex), and severe articular cartilage and joint disease. Although enzyme replacement therapy (ERT) is currently available for three of the mucopolysaccharidoses (MPS), there have been limited effects on bone and cartilage. Thus, new treatment approaches are clearly needed for these tissues, alone or as adjuncts to ERT.

Previous studies in MPS animal models showed that inflammation is a critical aspect of these disorders, secondary to GAG accumulation [14,15,17]. As part of

*Address for correspondence: Calogera M. Simonaro, PhD, Associate Professor, Department of Genetics \& Genomic Sciences, Mount Sinai School of Medicine, 1425 Madison Avenue, New York, NY 10029, USA. Tel.: +1 212659 6738; Fax: +1 212849 2447; E-mail: calogera.simonaro@mssm.edu. this inflammatory cascade, tumor necrosis factor-alpha (TNF- $\alpha$ ) and other inflammatory cytokines [e.g., interleukin (IL-1 $\beta$ ) are released from chondrocytes, resulting in apoptosis. In addition, matrix metalloproteinases (MMPs) are released, contributing to the joint and bone destruction. Lastly, due to the enhanced chondrocyte cell death, there is also a proliferation of immature chondrocytes, leading to abnormal matrix formation.

Signaling through toll-like receptors (TLRs) plays an important role in many autoimmune and inflammatory diseases, including rheumatoid arthritis (RA). Bacterial lipopolysaccharide (LPS), and more recently GAGs themselves, has been shown to activate one specific TLR, TLR4 [19]. The mechanism of LPS signaling through TLR4, in particular, has been extensively studied [20]. LPS forms a complex with a number of molecules, including LPS binding protein (LBP), which binds to TLR4 and initiates a cascade of events leading to the activation of the NF-kB transcription factor. This, in turn, promotes the production of pro-inflammatory cytokines (e.g., TNF- $\alpha$, IL-1 $\beta$ ), chemokines (e.g., macrophage inflammatory protein$1 \alpha$, macrophage inflammatory protein- $1 \alpha$ ), and MMPs (e.g., MMP-13). In addition to extrinsic activation by LPS, joint and bone trauma can release small molecular weight GAG fragments from the extracellular matrix, thus presenting them as intrinsic signals of inflammation via activation of TLR4 [19]. 
A direct consequence of TLR4 activation by either pathway (extrinsic or intrinsic) is TNF- $\alpha$ release. Among other things, MMP elevation is directly related to elevated TNF- $\alpha$, and this cytokine has been shown to promote cartilage degradation by stimulating the synthesis and release of these degradative proteases [10]. Previous studies have indicated that MMP-13 (collagenase 3) and MMP-1 are elevated in MPS synovial membranes, contributing to the pathologic remodeling of the extracellular matrix in these diseases, and potentially producing GAG fragments that activate TLR4 signaling. This concept was recently supported by the findings of Ausseil et al. [3], who demonstrated priming of microglia via the TLR4 pathway in the brains of MPS IIIB mice. We have also suggested that structural homologies between the oligosaccharide components of LPS and GAGs may contribute to TLR4 activation.

Gene and protein expression patterns have been assessed in synovial fibroblasts obtained from rats with MPS VI, revealing a markedly abnormal, proinflammatory expression pattern $[15,16]$. Of note, several molecules important for TLR4 signaling were shown to be elevated in the MPS cells (e.g., LBP, TLR4 itself, CD14, MyD88). GAG storage influences the levels of two important signaling lipids, ceramide and sphingosine-1-phosphate, known mediators of LPS activation. GAG treatment of chondrocytes led to elevation of the "pro-apoptotic" lipid, ceramide, consistent with enhanced apoptosis in these cells [14]. In contrast, a decrease of ceramide and elevated production of the "pro-survival" lipid, S1P, was observed in MPS synovial cells, leading to an enhanced proliferation rate and likely contributing to the hyperplastic MPS synovial membranes [16].

We further studied the effects of elevated TNF- $\alpha$ expression on MPS joint and bone pathogenesis. TNF- $\alpha$ and receptor activator of NF-kB ligand (RANKL) stimulate osteoclast differentiation in rheumatoid arthritis [13]. RANKL, essential for osteoclast differentiation, is expressed on $\mathrm{T}$ cells and fibroblasts within inflamed synovial tissue, and is regulated by proinflammatory cytokines. We found that RANKL expression and activity were markedly elevated in MPS synovial tissues and bone marrow. In addition, MPS bone marrow cultures had TRAP-positive multinucleated osteoclast-like cells (MNCs), consistent with the osteopenia previously observed in animal studies [12].

In order to develop "proof-of-concept" for the hypothesis regarding activation of the TLR4 signaling pathway in MPS disorders, MPS VII mice were crossed to TLR4 knockout mice to create double knockout
(DKO) animals [17]. Inactivation of TLR4 in MPS VII mice had a significant, positive effect on their growth. Quantitative analysis similarly revealed longer and thinner faces in the DKOs when compared to MPS VII. MicroCT revealed longer femora in the DKO compared to age- and gender-matched MPS VII mice, despite the fact that an osteopetrotic phenotype was still observed and that bone density was unchanged in the DKO. The growth plates of the DKO mice also were thinner and more organized when compared to MPS VII mice. However, despite these improvements, DKO mice still retained storage cells in the growth plates, which may be expected as the increased size of these MPS cells is due to GAG storage, which would not be affected by TLR4 KO.

Metcalf et al. [9] have shown that growth plates of MPS VII mice abnormally express mRNAs encoding several STAT transcription factors that are also selectively altered by inflammation. In particular, p-STAT3-Tyr705, a pro-proliferative factor, was decreased in MPS VII mice, and p-STAT1-Ser727, an anti-proliferative factor, was elevated. We assessed the protein levels of phosphorylated STAT1 and 3 in articular chondrocytes from the DKO mice, and found that they were normal, indicating that inactivation of the TLR4 pathway in MPS VII mice corrected the abnormal pSTAT1 and 3 expression. Similar alterations in STAT1 and 3 have been observed in RA, and have been attributed to activation of TNF- $\alpha$ [7,21]. We therefore measured serum TNF- $\alpha$ in MPS VII mice, and found an almost three-fold increase above normal. Of note, the elevated serum TNF- $\alpha$ was normalized in the DKO mice.

We have therefore established an important role of TLR4 in the pathogenesis of MPS bone and joint disease, and shown that inhibition of this pathway has significant, positive effects. Currently there are no FDAapproved drugs that directly target TLR4, although Remicade $^{\mathrm{TM}}$, which targets the downstream mediator, TNF- $\alpha$, has been approved for use in RA and other inflammatory diseases $[8,18,22]$. TNF- $\alpha$ is at the top of a cascade of proinflammatory cytokine production, and we hypothesized that its inhibition should prevent or slow the release of subsequent proinflammatory molecules, and reduce inflammation in the MPS joints. To date, Remicade ${ }^{\mathrm{TM}}$ treatment has not been systematically evaluated in MPS patients or animals.

MPS VI rats were therefore subjected to treatment with Remicade ${ }^{\mathrm{TM}}$. Importantly, in presymptomatic (1month-old) MPS VI animals, Remicade ${ }^{\mathrm{TM}}$ prevented the elevation of circulating TNF- $\alpha$, while in animals 
with advanced disease (6-month-old) the levels were reduced to normal. A similar response was seen when serum RANKL levels were determined, indicating that inhibition of TNF- $\alpha$ was having positive downstream effects [16]. The appearance, weight, and length of the treated MPS VI rats were not significantly different from untreated animals. This was not unexpected, as anti-TNF- $\alpha$ therapy alone was not predicted to improve bone growth. This is consistent with the fact that the stored GAGs and GAG fragments in the growth plate cells were not being reduced, and/or that the growth plate changes in rat may already be irreversible after 1 month of age. Further, Remicade ${ }^{\mathrm{TM}}$ inhibits TNF- $\alpha$ in the serum, and the growth plate is an extremely avascular tissue. However, TUNEL staining of the articular cartilage in the treated rats showed a 50\% reduction in the apoptotic index compared to untreated MPS VI animals, and there was less invasion of the synovial tissue into the underlying bone in the treated animals. These changes should lead to improved mobility, reduced pain, and other positive clinical effects.

In conclusion, GAG storage in MPS induces a complex sequence of molecular abnormalities leading to inflammation, apoptosis [cartilage], and hyperplasia [synovial membranes], resulting in poorly organized and metabolically abnormal connective tissue matrices [14-16]. Activation of the TLR4 pathway in the MPS disorders, likely a direct consequence of GAG storage, has major pathological effects on the joints and bones. Inactivation of this pathway in MPS VII/TLR4 DKO mice corrects many biochemical and clinical features of the disease, suggesting that drugs targeting this pathway could be effective in the treatment of these disorders. TLR4 activation leads to the elevation of TNF$\alpha$, and Remicade ${ }^{\mathrm{TM}}$, an FDA-approved anti-TNF- $\alpha$ drug, attenuated the inflammatory response in MPS VI animals, and led to improved joint pathology. We therefore propose that this and related anti-inflammatory treatments should be evaluated in MPS patients, alone or in conjuction with ERT. In addition to their direct effects on MPS pathology, such drugs also may, by reducing inflammation, increase the accessibility of synovial tissues to recombinant proteins, and improve the efficacy of ERT.

\section{Acknowledgements}

Supported by grants from the NIH [DK 25759, RR02512], The National MPS Society, and The Isaac Foundation. The author thanks Dr. Edward H. Schuch- man for support and assistance with these studies, and Ms. Yi Ge for her expert technical assistance. This commentary was developed as the result of a meeting of experts entitled "Promoting Bone Health in MPS VI: Framing New Therapies" held in Oakland, California in October, 2008. This meeting was supported by an educational grant from BioMarin Pharmaceutical, Inc., Novato, CA. BioMarin had no role in the content presented and discussed at the meeting.

\section{Conflict of interest}

Dr. Simonaro has no conflict of interest to report.

\section{References}

[1] A. Alonso-Ruiz, J.I. Pijoan et al., Tumor necrosis factor a drugs in rheumatoid arthritis: systematic review and metaanalysis of efficacy and safety, BMC Musculoskelet Disord 9 (2008), 52-63.

[2] D. Auclair, L.K. Hein et al., Intra-articular enzyme administration for joint disease in feline mucopolysaccharidosis VI: enzyme dose and interval, Pediatr Res 59 (2006), 538-543.

[3] J. Ausseil, N. Desmaris et al., Early neurodegeneration progresses independently of microglial activation by heparan sulfate in the brain of Mucopolysaccharidosis IIIB mice, PLoS ONE 3 (2008), e2296.

[4] M.K. Boettger, S. Hensellek et al., Antinociceptive effects of tumor necrosis factor alpha neutralization in a rat model of antigen-induced arthritis: evidence of a neuronal, Arthritis Rheum 58 (2008), 2368-2378.

[5] S.R. Goldring, Pathogenesis of bone erosions in rheumatoid arthritis, Curr Opin Rheumatol 4 (2002), 406-410.

[6] B.A. Hemmings, Akt signaling: linking membrane events to life and death decisions, Science 275 (1997), 628-630.

[7] X. Hu, J. Chen et al., Crosstalk among Jak-STAT, Toll-like receptor, and ITAM-dependent pathways in macrophage activation, Leukoc Biol 82 (2006), 237-243.

[8] D. Laharie, E. Chanteloup et al., Tolerance and efficacy of a postponed retreatment with infliximab in Crohn's disease primary responders, Aliment Pharmacol Ther 29 (2009), 12401248.

[9] J.A. Metcalf, Y. Zhang et al., Mechanism of shortened bones in mucopolysaccharidosis VII, Mol Genet Metab 97 (2009), 202-211.

[10] K. Migita, K. Eguchi et al., TNF-a mediated expression of membrane-type matrix metalloproteinase in rheumatoid synovial fibroblasts, Immunol 89 (1996), 553-557.

[11] E.F. Neufeld and J. Muenzer, The Metabolic and Molecular Bases of Inherited Disease, McGraw-Hill, New York, (2001), 3421-3452.

[12] J.D. Nutall, L.K. Brumfield et al., Histomorphometric analysis of the tibial growth plate in a feline model of mucopolysaccharidosis type VI, Calcif Tissue Int 65 (1999), 47-52.

[13] Y. Shigeyama, T. Pap et al., Expression of osteoclast differentiation factor in rheumatoid arthritis, Arthritis Rheum 43 (2000), 2523-2530. 
[14] C.M. Simonaro, M.E. Haskins et al., Articular chondrocytes from animals with a dermatan sulfate storage disease undergo a high rate of apoptosis and release nitric oxide and inflammatory cytokines: a possible mechanism underlying degenerative joint disease in the mucopolysaccharidoses, Lab Invest 81 (2001), 1319-1328.

[15] C.M. Simonaro, M. D'Angelo et al., Joint and bone disease in mucopolysaccharidoses VI and VII: identification of new therapeutic targets and biomarkers using animal models, Pediatric Res 57 (2005), 701-707.

[16] C.M. Simonaro, M. D'Angelo et al., Mechanism of glycosaminoglycan-mediated bone and joint disease: implications for the mucopolysaccharidoses and other connective tissue diseases, Am J Pathol 172 (2008), 112-122.

[17] C.M. Simonaro, Y. Ge et al., Involvement of the Toll-like receptor 4 pathway and use of TNF-alpha antagonists for treatment of the mucopolysaccharidoses, Proc Natl Acad Sci U S A Dec 14 (2009). [Epub ahead of print].

[18] V. Strand and J.A. Singh, Improved health-related quality of life with effective disease-modifying antirheumatic drugs: evidence from randomized controlled trials, Am J Manag Care 13 (2006), S237-S252.

[19] K.R. Taylor, Y. Yamasaki et al., Recognition of hyaluronan released in sterile injury involves a unique receptor complex dependent on Toll-like receptor 4, CD44, and MD-2, J Biol Chem 282 (2007), 18265-18275.

[20] M. Triantafilou and K. Triantafilou, Lipopolysaccaride recognition: CD14, TLRs and the LPS-activation cluster, Trends in Immuno 23 (2002), 301-304.

[21] J.G. Walker, M.J. Ahern et al., Expression of Jak3, STAT1, STAT4, and STAT6 in inflammatory arthritis: unique Jak3 and STAT4 expression in dendritic cells in seropositive rheumatoid arthritis, Ann Rheum Dis 65 (2006), 149-156.

[22] A.L. Weaver, Efficacy and safety of the anti-TNF biologic agents, Mod Rheumatol 14 (2004), 101-112.

[23] R.O. Williams and M. Feldmann, Anti-tumor necrosis factor ameliorates joint disease in murine collagen-induced arthritis, Proc Natl Acad Sci USA 89 (1992), 9784-9788. 